\title{
Observables and unobservables in dark energy cosmologies
}

\author{
Luca Amendola, ${ }^{1}$ Martin Kunz, ${ }^{2}$ Mariele Motta, ${ }^{1,3}$ Ippocratis D. Saltas, ${ }^{4}$ and Ignacy Sawicki ${ }^{1}$ \\ ${ }^{1}$ ITP, Ruprecht-Karls-Universität Heidelberg, Philosophenweg 16, 69120 Heidelberg, Germany \\ ${ }^{2}$ Département de Physique Théorique and Center for Astroparticle Physics, \\ Université de Genève, Quai E. Ansermet 24, CH-1211 Genève 4, Switzerland \\ ${ }^{3}$ Instituto de Física Gleb Wataghin - UNICAMP, 13083-970 Campinas, SP, Brazil \\ ${ }^{4}$ School of Physics 83 Astronomy, University of Nottingham, Nottingham, NG7 2RD, United Kingdom
}

(Dated: August 21, 2018)

\begin{abstract}
The aim of this paper is to answer the following two questions: (1) Given cosmological observations of the expansion history and linear perturbations in a range of redshifts and scales as precise as is required, which of the properties of dark energy could actually be reconstructed without imposing any parameterization? (2) Are these observables sufficient to rule out not just a particular dark energy model, but the entire general class of viable models comprising a single scalar field?

This paper bears both good and bad news. On one hand, we find that the goal of reconstructing dark energy models is fundamentally limited by the unobservability of the present values of the matter density $\Omega_{\mathrm{m} 0}$, the perturbation normalization $\sigma_{8}$ as well as the present matter power spectrum. On the other, we find that, under certain conditions, cosmological observations can nonetheless rule out the entire class of the most general single scalar-field models, i.e. those based on the Horndeski Lagrangian.
\end{abstract}

\section{INTRODUCTION}

Research in dark energy (DE) cosmology is generally devoted to building viable models and to constraining them from observations (see, for instance, the reviews $[1-3])$. The models are usually characterized by a small number of properties at background and linearperturbation level (equations of state, speeds of sound, masses, coupling strengths, etc.) which then have some particular effect on the phenomenology (evolution of the scale factor and perturbations of matter and DE itself). In this paper, we employ a different approach, aiming to answer the following questions:

1. Assuming the ideal case of cosmological observations of the expansion history and linear perturbations in a range of redshifts and scales which are as precise as is required, which physical properties (such as e.g. the Hubble rate $H(z)$, the perturbation normalization $\sigma_{8}$, the perturbation growth rate $f$, etc.) could actually be reconstructed if we were to refrain from any parameterization of dark energy?

2. Can we use these observable quantities to rule out not just some particular cosmological model but the entire class of viable single scalar-field models?

We are of course not the first to attempt to study dark energy cosmologies in a model-independent way. In Ref. [4] the author argues for a reconstruction method which does not rely even on Einstein equations. This method can in principle directly measure the space-time curvature but cannot test a modification of gravity. On background level, the power of observations to constrain the expansion history of the universe by assuming $\Lambda$-cold dark matter $(\Lambda \mathrm{CDM})$ as a null test was studied in Refs [5, 6], while a principle-component analysis of the equation of state constraints was developed in Ref. [7]. Recently, even the validity of the assumption of the Copernican principle was tested [8]. On the level of linear perturbations, parameterizations are usually used to limit the freedom in the model-independent description of growth of structure [9-13], although principle components analysis has also been employed [14]. Another way to limit the freedom in the fully general description is to exploit the structure which any general-relativity-like theory of dark energy must obey: such approaches were discussed in [1518]. All of the above approaches, when contrasted with data, require parameterizations in order to break degeneracies, but simultaneously introduce parameterizationdependent biases.

The difference of our approach is that, given the minimum of assumptions, we first elucidate the observables that measurements can in principle provide without the assumption of any dark energy model in particular. It is only then that we use these model-independent observables to construct tests which might eliminate or confirm particular models. Our approach is closest in spirit to Ref. [19], the results of which we extend.

In this paper, we completely ignore the practical problems and limitations of the observations and assume that good-enough statistics with sufficiently small systematic errors can be achieved in the range of redshifts and scales discussed here. By exploring this idealized case we try to discover the fundamental limits to which observations in a dark energy cosmology are subject.

We adopt metric signature $(-+++)$, while a comma denotes a partial derivative. We interchangeably use coordinate time $t$, scale factor $a, e$-foldings $N \equiv \ln a$ and the redshift $z$ as time variables. Overdots denote derivatives with respect to $t$, primes with respect to $N$. The subscript 0 denotes the present time. We also make use of the notation of $[20,21]$, to which we refer for a thorough study of linear perturbations in the context of the 
Horndeski theories.

\section{ASSUMPTIONS}

In keeping with the spirit of generality, we first wish to make the minimum of assumptions on the geometry and matter content of our Universe that will allow us to interpret observations at all. In the following we assume only that:

(a) The geometry of the Universe is well described by small linear perturbations living in an FLRW metric with scale factor $a(t)$. We will not consider possible observations of rotational perturbation modes nor of gravitational waves, as these are irrelevant for structure formation in late-time cosmology.

(b) The matter content (i.e. dark matter and baryonic matter) is pressureless or evolves in a known way.

(c) The relation between the galaxy distribution and the matter distribution at linear scales can be modeled as $\delta_{\text {gal }}=b(k, a) \delta_{\mathrm{m}}$, where $b(k, a)$ is the potentially scale- and time-dependent linear bias, while at the same time there is no bias between the velocities of galaxies and matter. This implies that both the baryonic and dark matter respond in the same way to the gravitational potentials and that the statistical velocity bias due to galaxies sampling preferentially over-dense regions [22] is negligible on the scales of interest.

(d) The late-time universe is effectively described by the action

$$
S=\int \mathrm{d}^{4} x \sqrt{-g}\left(\frac{1}{2} R+\mathcal{L}_{x}+\mathcal{L}_{\mathrm{m}}\right)
$$

(setting $8 \pi G_{\mathrm{N}}=1$ ) which includes the EinsteinHilbert term for the metric $g_{\mu \nu}$ and the Lagrangian $\mathcal{L}_{\mathrm{m}}$ describing pressureless matter fluids, both baryons and dark matter, between which we will not differentiate here. Any other terms are ascribed to the DE Lagrangian $\mathcal{L}_{x}$, which represents some consistent theory potentially depending on extra degrees of freedom or $g_{\mu \nu}$ (i.e. modifications of gravity). ${ }^{1}$ We will neglect the radiation component because all the observations are assumed to be performed well after decoupling. In nonminimally coupled models, the Lagrangian $\mathcal{L}_{\mathrm{m}}$ depends on a different metric, related to $g_{\mu \nu}$ through some transformation. Here we assume, however, that we have already reformulated the action so that matter moves on the geodesics of $g_{\mu \nu}$.

\footnotetext{
1 A consistent theory is understood here to be a theory free of ghost and other catastrophic instabilities that can in general occur in generalized gravity and dark energy models.
}

We employ the above minimal framework to address question (1) by considering the background observables in section III and those arising from linear perturbations in section IV. To answer question (2) we need another crucial assumption, concerning the degrees of freedom in the dark energy Lagrangian:

(e) The Lagrangian $\mathcal{L}_{x}$, which describes dark energy, is any one of the Lagrangians describing a single scalar field governed by second-order equations of motion. We call this scalar field dark energy, but we do not necessarily require it to be driving the current acceleration. For example, it could be that the late time acceleration is effectively driven by a cosmological constant, but in the presence of this additional degree of freedom. The assumption of a scalar field ensures that there are no gross violations of isotropy. The limitation to second order is a necessary condition to ensure that the model is not subject to instabilities (see e.g. [23]). ${ }^{2}$ We will therefore assume that the dark energy is governed by the most general Lagrangian which fulfills these requirements: $\frac{1}{2} R+\mathcal{L}_{x}$ will form the Horndeski Lagrangian (HL $[25,26]$ ). We dedicate section $\mathrm{V}$ to this system.

\section{BACKGROUND OBSERVABLES}

From assumptions $(a)-(c)$, by varying the action eq. (1) with respect to the metric, we obtain a Friedmann equation that can be written as

$$
H^{2}-H_{0}^{2} \Omega_{k 0} a^{-2}=\frac{1}{3}\left(\rho_{x}+\rho_{\mathrm{m}}\right)
$$

where $H_{0}$ is the present value of the Hubble parameter, $\Omega_{k 0}$ the present curvature density parameter and $\rho_{\mathrm{m}}$ is the matter energy density. From assumption $(b), \rho_{\mathrm{m}}$ evolves as $a^{-3}$, and $\rho_{x}$ is the energy density of the terms coming from $\mathcal{L}_{x}$.

Observations of the cosmic expansion are essentially estimations of distances $D(z)$ (i.e. luminosity or angulardiameter distances) or directly $H(z)$ (e.g. using measurements of longitudinal baryon acoustic oscillations, or real-time redshift-drift observations [27]) based on the existence of standard candles, rods or clocks. More exactly, standard candles or rods measure $H(z)$ up to a multiplicative constant, related to the unknown absolute measure of the source luminosity or proper length. For instance, the flux of supernovae Ia (SNIa) with absolute luminosity $L$ are known only up to the constant $L H_{0}^{2}$; only ratios of fluxes at different redshifts are independent

2 This class of Lagrangians includes such theories as $f(R)$ gravity, despite their naively fourth-order equations of motion. This is because we can always introduce the a priori hidden scalar explicitly through a Legendre transformation [24]. 
of the absolute normalization. The same is true of baryon acoustic oscillations: they measure only the ratio of the sound horizon at last scattering and the Hubble radius $H^{-1}(z)$. We can therefore say that, without additional assumptions, background cosmological observations estimate $D(z)$ up to an overall constant as well as the dimensionless Hubble function $E(z) \equiv H(z) / H_{0}$. Notice, however, that real-time redshift-drift observations can estimate the absolute value of $H(z)$, while local measurements of the expansion measure $H_{0}$.

Combining $D(z)$ with $H(z)$, we can also estimate the present curvature parameter $\Omega_{k 0}$. We can therefore determine the evolution of the combined matter and dark energy content, $1-\Omega_{k}$, at all times. If we assume that there are only two components of the cosmic fluid then we have only one free parameter, $\Omega_{\mathrm{m} 0}$. In fact, we can write

$$
\Omega_{x}=1-\Omega_{k}-\Omega_{\mathrm{m}}=1-\frac{1}{E^{2}}\left(\Omega_{k 0} a^{-2}+\Omega_{\mathrm{m} 0} a^{-3}\right) .
$$

Therefore, we conclude that from background observables we can reconstruct both $\Omega_{\mathrm{m}}$ and $\Omega_{x}$, but only up to $\Omega_{\mathrm{m} 0}$ [28], since one can compensate for any change of $\Omega_{\mathrm{m} 0}$ with a modification of the DE model. Of course, if we parameterize the evolution of $\Omega_{x}$ with a simple equation of state, we can break the degeneracy with $\Omega_{\mathrm{m} 0}$, as is usually done in analyses of SNIa data, but that is exactly what we are trying to avoid in this work.

The same result is valid if instead of pure pressureless matter one includes further components (e.g. massive neutrinos) that evolve with an effective equation of state $w_{\mathrm{m}}(z)$, provided $w_{\mathrm{m}}(z)$ can be inferred from other observations (e.g. knowledge of the neutrino masses).

\section{LINEAR PERTURBATION OBSERVABLES}

The linear perturbation observables are the correlations of positions, velocities and shapes (ellipticities) of sources (i.e. galaxies, Lyman- $\alpha$ lines, clusters, background radiation) in angular separation and redshift. Given knowledge of $D(z)$, these can be converted to the more usual dependence on wavenumber $k$ and redshift.

Let us first discuss the clustering of matter. We denote the root mean square of the correlation of galaxy number counts in Fourier space as $\delta_{\text {gal }}$ (i.e. $\delta_{\text {gal }} \equiv P_{\text {gal }}^{1 / 2}(k, z)$ where $P_{\text {gal }}$ is the galaxy power spectrum). We define from now on the wavenumber $k$ to be the physical wavenumber expressed in the units of the cosmological horizon, i.e. $k=k_{\text {phys }} / a H$ ( $k$ is independent of $H_{0}$ if $k_{\text {phys }}$ is measured in $\left.h / \mathrm{Mpc}\right)$. This means that $k$ is time-dependent. We observe galaxies, not matter perturbations, so as anticipated we need to introduce a bias function $b(k, z)$ such that $\delta_{\text {gal }}=b \delta_{\mathrm{m}}$. Without the assumption of a particular model, DE perturbations are unknown. In many models they are not at all small compared to matter perturbations. For this reason, we will define the total density perturbation $\delta_{\mathrm{t}} \equiv \Omega_{\mathrm{m}} \delta_{\mathrm{m}}+\Omega_{x} \delta_{x}$ and introduce the bias $B$ of galaxies with respect to it,

$$
\delta_{\text {gal }}=B \delta_{\mathrm{t}}=B Z \Omega_{\mathrm{m}} \delta_{\mathrm{m}}
$$

where $Z(k, a) \equiv 1+\Omega_{x} \delta_{x} /\left(\Omega_{\mathrm{m}} \delta_{\mathrm{m}}\right)$ is a function of space and time that depends on the clustering of the $x$-component. Then we have that $b=B Z \Omega_{\mathrm{m}}$.

Let us denote the initial total density perturbation spectrum at decoupling as $\delta_{\mathrm{t}, \text { in }}^{2}(k)$ and as $G_{\mathrm{t}}(k, z)$ the scale-dependent growth function of the linear total density perturbations, normalized to unity at present. If the galaxies move with the same velocity field as matter, the galaxy velocity divergence $\theta_{\text {gal }}$ in the sub-Hubble regime is related to the matter density perturbation as $\theta_{\text {gal }}=\theta_{\mathrm{m}}=-\delta_{\mathrm{m}}^{\prime}=-f \delta_{\mathrm{m}}$, by the continuity equation for matter. We then obtain $\theta_{\text {gal }}=-(f / b) \delta_{\text {gal }}$, where $f=G^{\prime} / G$ is the linear matter growth rate and $G(k, z)$ is the growth function for matter perturbations, both of which are scale-dependent for general DE models. This velocity field generates redshift distortions as a function of the direction cosine $\mu=(\vec{k} \cdot \vec{\ell}) / k$ where $\vec{\ell}$ is the unit line-of-sight vector. The observable $\delta_{\text {gal }}$ can therefore be expressed as [29]

$$
\delta_{\text {gal }}(k, z, \mu)=G_{\mathrm{t}} B \sigma_{8, \mathrm{t}}\left(1+\frac{f}{b} \mu^{2}\right) \delta_{\mathrm{t}, 0}(k),
$$

where $\sigma_{8, \mathrm{t}}$ is the present normalization of the total density spectrum. Now we can write $G_{\mathrm{t}} B=$ $Z \Omega_{\mathrm{m}} \delta_{\mathrm{m}} B /\left(Z \Omega_{\mathrm{m}} \delta_{\mathrm{m}}\right)_{0}=G b /\left(Z \Omega_{\mathrm{m}}\right)_{0}$, so we have the almost-standard expression

$$
\delta_{\text {gal }}(k, z, \mu)=G b \sigma_{8}\left(1+\frac{f}{b} \mu^{2}\right) \delta_{\mathrm{t}, 0}(k) .
$$

In this expression we set, using the $8 \mathrm{Mpc} / h$ spherical Fourier space window function $W(k)$,

$$
\sigma_{8}=\frac{\sigma_{8, \mathrm{t}}}{\left(Z \Omega_{\mathrm{m}}\right)_{0}}=Z_{0}^{-1}\left(\int Z_{0}^{2} P_{\mathrm{m} 0} W^{2}\left(k, R_{8}\right) \mathrm{d} k\right)^{1 / 2}
$$

which is equivalent to the usual normalization $\sigma_{8}$ if $Z$ depends weakly on $k$.

To discuss weak lensing, we introduce the standard perturbed metric in longitudinal gauge $\mathrm{d} s^{2}=-(1+$ $2 \Psi) \mathrm{d} t^{2}+a^{2}(1+2 \Phi) \delta_{i j} \mathrm{~d} x^{i} \mathrm{~d} x^{j}$. Since later we will confine ourselves to a scalar dark energy, as part of assumption $(a)$, we assume that the DE does not excite vector and tensor modes so that only scalar modes need to be included. It is helpful to introduce the function $Y$, the effective gravitational constant for matter, and the anisotropic stress $\eta$ defined as (see e.g. $[12,21]$ )

$$
Y(k, a)=-\frac{2 k^{2} \Psi}{3 \Omega_{\mathrm{m}} \delta_{\mathrm{m}}}, \quad \eta(k, a)=-\frac{\Phi}{\Psi} .
$$

Both $\eta$ and $Y$ are unity on sub-Hubble scales if $\mathcal{L}_{x}$ is a constant (i.e. the DE is a cosmological constant) and the matter is a perfect fluid. In the linear regime, the lensing 
effect is proportional to the lensing potential, which itself is driven by the density perturbations (see e.g. [30]). In general, this relation can be written as

$$
\begin{aligned}
k^{2} \Phi_{\text {lens }} & =k^{2}(\Psi-\Phi)=-\frac{3}{2} Y(1+\eta) \Omega_{\mathrm{m}} \delta_{\mathrm{m}} \\
& =-\frac{3}{2} Y(1+\eta) \frac{G_{\mathrm{t}} \sigma_{8, \mathrm{t}} \delta_{\mathrm{t}, 0}}{Z}=-\frac{3}{2} \Sigma G \Omega_{\mathrm{m}} \sigma_{8} \delta_{\mathrm{t}, 0},
\end{aligned}
$$

where we have defined the "modified lensing" function $\Sigma(k, z) \equiv Y(1+\eta)$. The ellipticity correlation is an integral function of $\Phi_{\text {lens }}$ within a window function that depends on the survey geometry (see e.g. [19]). Assuming good-enough knowledge of the galaxy distribution one can differentiate the correlation integral and obtain the quantity

$$
\sigma(k, z) \equiv \frac{2}{3}\left(k^{4} P_{\Phi_{\text {lens }}}\right)^{1 / 2}=\frac{1}{a^{3} E^{2}} \Omega_{\mathrm{m} 0} \Sigma G \sigma_{8} \delta_{\mathrm{t}, 0} .
$$

Then from $\delta_{\text {gal }}(k, z, \mu)$ (with e.g. $\mu=0,1$ ) and $\sigma(k, z)$ one can measure the three quantities $A, R, L$ defined as

$$
\begin{aligned}
A & =G b \sigma_{8} \delta_{\mathrm{t}, 0}, \quad R=G f \sigma_{8} \delta_{\mathrm{t}, 0}, \\
L & =\Omega_{\mathrm{m} 0} \Sigma G \sigma_{8} \delta_{\mathrm{t}, 0} .
\end{aligned}
$$

The quantities that connect the observations to theory (i.e. to the Lagrangian $\mathcal{L}_{x}$ ) are $\Omega_{x}, f, \Sigma$, so it would be optimal to estimate them directly from observations. Now, the cosmic microwave background anisotropy allows one to measure, at least in principle, the initial potential $\Psi_{\text {in }}$ through the Sachs-Wolfe effect. It is, however, impossible to derive from this information the present power spectrum $\delta_{\mathrm{t}, 0}$ since it also depends on a scale- and time-dependent transfer function. Absent a model for DE, this transfer function is unknown and since it acts to process the total perturbation spectrum, changing its $k$-dependence, it also makes $\delta_{\mathrm{t}, 0}$ an unknown without further assumptions. This argument shows that the only

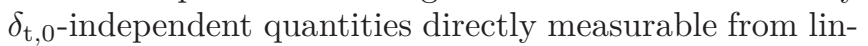
ear cosmological observations are ratios of $A, R, L$, and their $N$-derivatives, i.e.

$$
\begin{aligned}
& P_{1} \equiv R / A=f / b, \\
& P_{2} \equiv L / R=\Omega_{\mathrm{m} 0} \Sigma / f, \\
& P_{3} \equiv R^{\prime} / R=f+f^{\prime} / f .
\end{aligned}
$$

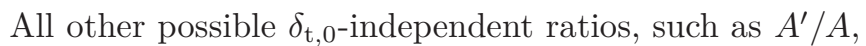
$L^{\prime} / L$ or $R^{\prime} / L$ or higher-order $N$-derivatives, can be obtained as combinations of $P_{1-3}$ and their derivatives, for instance $L^{\prime} / L=P_{2}^{\prime} / P_{2}+P_{3}$. Other linear-perturbation probes, such as integrated Sachs-Wolfe, cross-correlations or 21-cm flux measurements, add statistics and might extend the observational range but do not break the fundamental degeneracy.

The quantity $P_{1}$ is a well-known observable quantity, often denoted $\beta$ [30]; since it involves the bias function $b$, related in an unknown way to the model of dark energy, we will not consider it any longer in this paper. The quantity $P_{2}$ has already been introduced in [19] as
$E_{G}$ as a test of modified gravity, but the fact that $\Omega_{\mathrm{m} 0}$ is not an observable was not discussed there. The quantity $R$ contains the term $G f \sigma_{8}$, also denoted as $f \sigma_{8}(z)$ in the literature [31]. This term is often considered to be a directly observable quantity, but, as we have argued, this is only true if one assumes a model for DE, or at least a parametrized form of $\delta_{\mathrm{t}, 0}$; otherwise, the modelindependent observable combination is $P_{3}=R^{\prime} / R$. It is important to realize that even a perfect knowledge of $P_{3}$ does not imply knowledge of $f$ since the equation $f^{\prime} / f+f=P_{3}(k, z)$ cannot be solved without the unknown $k$-dependent initial condition for $f$. Finally, notice that we did not need to assume Gaussian fluctuations nor isotropy of the power spectrum.

Measurements of galaxy peculiar velocities and their time derivative directly estimate $\Psi$ through the Euler equation, which would give the quantity $V=$ $\Omega_{\mathrm{m} 0} Y G \sigma_{8} \delta_{\mathrm{t}, 0}$. Then one can form the observable, $L / V=1+\eta$, which measures the anisotropic stress. This new observable is not independent since it can be written in terms of $E, P_{2}, P_{3}$ (see Eq. (21)). Moreover, the estimation of $V$ requires a delicate subtraction of the peculiar redshift from the cosmological redshift by using distance indicators such as Cepheids and therefore a number of additional assumptions on the source physics. No current or foreseeable method to estimate the peculiar velocity field (let alone its derivative) has been shown to be reliable beyond a few hundred megaparsecs (see e.g. Ref. [32]), so we will not pursue this possibility any further in this paper.

Our first result is that linear cosmological observations can at best determine only $E \equiv H / H_{0}$ as function of time (as well as $\Omega_{k 0}$ but not $\Omega_{\mathrm{m} 0}$ ) and the observable combinations $P_{1-3}$ as functions of time and space, within the range of the observations themselves. To achieve this, we need to combine galaxy clustering and weak lensing in the same redshift range. It is possible that one can determine other combinations from non-linear effects, but this will certainly introduce new uncertainties (e.g. a non-linear bias).

\section{THE HORNDESKI LAGRANGIAN}

It is now time to use also assumption (e) regarding the explicit form of the dark energy Lagrangian. In the choice of the action (1), we have assumed that all matter components (i.e. dark matter and baryons) feel the same gravitational force and propagate on geodesics of the metric $g_{\mu \nu}$. In addition, we now explicitly assume that the DE is modeled by a single scalar field $\phi$ described by the Horndeski Lagrangian (HL). The HL is defined as the sum of four terms $\mathcal{L}_{2}$ to $\mathcal{L}_{5}$ that are fully specified by a non-canonical kinetic term $K(\phi, X)$ and three in principle arbitrary coupling functions $G_{3,4,5}(\phi, X)$, where $X=-g_{\mu \nu} \phi^{, \mu} \phi^{, \nu} / 2$ is the canonical kinetic term, 


$$
\begin{aligned}
\mathcal{L}_{2}= & K(\phi, X), \\
\mathcal{L}_{3}= & -G_{3}(\phi, X) \square \phi, \\
\mathcal{L}_{4}= & G_{4}(\phi, X) R+G_{4, X}\left[(\square \phi)^{2}-\left(\nabla_{\mu} \nabla_{\nu} \phi\right)^{2}\right], \\
\mathcal{L}_{5}= & G_{5}(\phi, X) G_{\mu \nu} \nabla^{\mu} \nabla^{\nu} \phi-\frac{G_{5, X}}{6}\left[(\square \phi)^{3}-\right. \\
& \left.-3(\square \phi)\left(\nabla_{\mu} \nabla_{\nu} \phi\right)^{2}+2\left(\nabla_{\mu} \nabla_{\nu} \phi\right)^{3}\right] .
\end{aligned}
$$

The Horndeski Lagrangian is the most general Lagrangian for a single scalar which gives second-order equations of motion for both the scalar and the metric on an arbitrary background. This is a necessary, but not sufficient, condition for the absence of ghosts. ${ }^{3}$ In general, the equation of motion for the scalar will couple it to the matter energy density. The metric potentials $\Phi$ and $\Psi$ are as usual determined by the Poisson and anisotropy equations, which are constraints, and therefore do not have independent dynamics. We note that the generalization to the case of multiple scalar fields has been discussed in Ref. [33].

In what follows, we will assume that the so-called quasi-static limit is valid for the evolution of perturbations. This implies that we are observing scales significantly inside the cosmological horizon, $k \equiv$ $k_{\text {phys }} /(a H) \gg 1$, and inside the Jeans length of the scalar, $c_{\mathrm{s}} k \gg 1$, such that the terms containing $k$ dominate over the time-derivative terms. The sound speed $c_{\mathrm{S}}$ is a particular function of the HL functions $K, G_{3-5}$ evaluated at the background level and we have presented it in Eq. (A4) [20]. In this quasi-static limit for a model belonging to the HL, one obtains [21]

$$
\eta=h_{2}\left(\frac{1+k^{2} h_{4}}{1+k^{2} h_{5}}\right), Y=h_{1}\left(\frac{1+k^{2} h_{5}}{1+k^{2} h_{3}}\right) .
$$

for suitably defined functions $h_{1-5}$. In this limit, one also has $Z=Y \eta$.

The functions $h_{1-5}$ express the modification of gravity induced by the HL. In real space they induce a timedependent Yukawa correction to the Newtonian potential. They are all combinations of HL functions $K, G_{3,4,5}$ and their derivatives with respect to $\phi$ and $X$, all evaluated on the background and are therefore time- but not $k$-dependent,

$$
h_{i} \equiv h_{i}(z) \equiv h_{i}(\phi, X)
$$

The explicit expressions for the functions $h_{i}$ are very complicated and not particularly illuminating; we have nonetheless presented them in appendix A. For $\Lambda \mathrm{CDM}$ one has simply $h_{1,2}=1$ and $h_{3,4,5}=0$. If the two

3 The constraints on the HL arising from stability considerations are derived in Ref. [20]. We have also presented them in appendix A. gravity-coupling functions in the $\mathrm{HL}, G_{4}, G_{5}$, are constant, i.e. the effective Planck mass is constant, then $\eta=1$; if moreover $G_{3}$ depends only on $\phi$ (i.e. k-essence), then also $Y=1$ and there are no modified-gravity effects at all in this quasi-static limit.

It is worth noting that one could have arrived at the form of Eqs (17) given just our assumptions of secondorder equations of motion, the symmetries of the FRW background and quasi-staticity.

In the same quasi-static limit, from the matter conservation equation, we obtain

$$
\delta_{\mathrm{m}}^{\prime \prime}+\left(2+\frac{H^{\prime}}{H}\right) \delta_{\mathrm{m}}^{\prime}=-k^{2} \Psi=\frac{3}{2} \Omega_{\mathrm{m}} \delta_{\mathrm{m}} h_{1}\left(\frac{1+k^{2} h_{5}}{1+k^{2} h_{3}}\right),
$$

or

$$
f^{\prime}+f^{2}+\left(2+\frac{H^{\prime}}{H}\right) f=\frac{3}{2} \Omega_{\mathrm{m}} h_{1}\left(\frac{1+k^{2} h_{5}}{1+k^{2} h_{3}}\right) .
$$

On the other hand, we can write for the weak-lensing function $\Sigma$,

$$
\Sigma=Y(1+\eta)=h_{6}\left(\frac{1+k^{2} h_{7}}{1+k^{2} h_{3}}\right),
$$

where we have introduced two auxiliary functions, $h_{6}=$ $h_{1}\left(1+h_{2}\right)$ and $h_{7}=\left(h_{5}+h_{4} h_{2}\right) /\left(1+h_{2}\right)$. As an aside, one can show that if $G_{3}, G_{4}$ depend only on $\phi$ and $G_{5}=$ const, i.e. we are dealing with a k-essence theory nonminimally coupled to gravity, then $h_{2}=1$ and $h_{7}=h_{3}$ so that $\Sigma$ becomes independent of $k$. In this limit, the gravitational potential felt by photons is not distorted (we have discussed this model in detail in Ref. [34]).

From the observables $P_{i}, E$ we can construct a modelindependent relation measuring the anisotropic stress $\eta$ as follows. From $P_{2}, P_{3}$ we can obtain $f=\Omega_{\mathrm{m} 0} \Sigma / P_{2}$ and $f^{\prime}=P_{3} \Omega_{\mathrm{m} 0} \Sigma / P_{2}-\left(\Omega_{\mathrm{m} 0} \Sigma / P_{2}\right)^{2}$. Inserting this in Eq. (19) and employing Eq. (20), after a little algebra we obtain a simple relation.

$$
\frac{3 P_{2}(1+z)^{3}}{2 E^{2}\left(P_{3}+2+\frac{E^{\prime}}{E}\right)}-1=\eta=h_{2}\left(\frac{1+k^{2} h_{4}}{1+k^{2} h_{5}}\right) .
$$

It is important to stress that the 1.h.s. of Eq. (21) is a function of model-independent observables, and thus a model-independent measurement of $\eta$, valid under our assumptions $(a)-(d)$, but not requiring $(e)$. The form of the last term is determined by the QS limit of the Horndeski Lagrangian. Given the above, we can exclude all dark energy models described by a single scalar field in the QS limit by showing that the anisotropic stress measured from the observation data does not follow the particular scale dependence mandated by Eq. (21). Equation (21) must be valid in fact at all times and scales where the quasi-static limit is valid. At any given epoch $z^{*}$, this equation involves the three unknowns $h_{2}, h_{4}, h_{5}$ all evaluated at $z^{*}$. If at this epoch we observe $E$ and $P_{2}, P_{3}$ at more than three $k$-modes, we can form an overconstrained system. If for any $z^{*}$ this system has no 
solution then the observations are inconsistent with the quasi-static limit of HL. Equivalently, from Eq. (21) one can obtain a consistency relation that depends only on observable quantities. Defining $g(z, k) \equiv \frac{\left(R E a^{2}\right)^{\prime}}{L E a^{2}}$, one has in fact

$$
2 g^{(1)} g^{(3)}-3\left(g^{(2)}\right)^{2}=0,
$$

where $g^{(n)}$ is the $n$-th derivative of $g$ with respect to $k^{2}$. If this condition fails at any one redshift, the DE is not described by the HL in the linear quasi-static limit. This is the second main result of this paper. Needless to say, a cosmological constant satisfies this consistency relation.

On the other hand, if there are consistent solutions then we obtain an indication in favor of the HL and also direct constraints on it. For instance, if observationally we find that $P_{2}, P_{3}$ do not depend on $k$, then from Eq. (21) we see that the condition $h_{4}=h_{5}$ must be satisfied.

If the consistency relation is not satisfied, the only possible way out of our conclusion is that the conditions for the linear quasi-static limit that we employed to derive Eq. (17) are not satisfied. This can occur if the rate of change of the functions $h_{1-5}$ is very large, e.g. if $h_{j}^{\prime} / h_{j} \approx c_{\mathrm{s}}^{2} k^{2} \gg 1$ for some $j$. However if the field $\phi$ drives the current accelerated expansion (this is indeed an additional assumption) we expect it to be slow rolling on time scales of the order of $\dot{h}_{j} / h_{j} \sim H$, i.e. $h_{j}^{\prime} / h_{j} \approx 1$. If the sound speed squared $c_{\mathrm{s}}^{2}$ is of order unity, then on typical astrophysical scales of $100 \mathrm{Mpc} / h$ one has $k^{2} \approx 10^{3}$, so the quasi-static limit should be very well satisfied. However if $c_{\mathrm{s}}^{2}$ is less than say $10^{-2}$ then the simple form of Eq. (17) is no longer valid. One might then expect oscillating terms in the $Y, \eta$ equations; it is possible that this behavior could be probed, and possibly rejected, by a similar method we are discussing here but a full analysis of this "cold dark energy" scenario would be required. Another potential difficulty is the fact that these more general scalar field theories contain non-linearities in principle independent of those in the matter perturbations. It may prove difficult to determine on which scales the linear approximation for the dark energy is valid, if at all

From $P_{2}$ and $P_{3}$ one can build other consistency equations, e.g. by differentiating $P_{2}$ or the combination $P_{2}^{\prime} / P_{2}+P_{3}=\Sigma^{\prime} / \Sigma+f$ with respect to $N$ and again employing Eqs (19), (20). These relations however require derivatives of the observables $P_{2}, P_{3}$ and will introduce derivatives of the $h_{1-5}$ functions, so appear to be less useful than Eq. (21).

We observe also that the propagation speed $c_{\mathrm{T}}$ of gravitational waves is a function of the HL coupling functions, see Eq. (A3) [35]. A detection of a source both in gravitational and electromagnetic waves could allow for a measurement of $c_{\mathrm{T}}$ and therefore new independent constraints on the HL [36].

\section{CONCLUSIONS}

We have shown that cosmological linear observations can measure only $\Omega_{k 0}, E \equiv H / H_{0}$ and the combinations $P_{1}=f / b, P_{2}=\Omega_{\mathrm{m} 0} \Sigma / f$ and $P_{3}=f+f^{\prime} / f$. Parameters such as $\Omega_{\mathrm{m} 0}, \sigma_{8}$ or functions such as $G f \sigma_{8}$ are not directly model-independent measurable quantities via linear cosmological observations alone. This limits in a fundamental way the knowledge of, among others, the evolution of the DE density parameter $\Omega_{x}$, its equation of state, or the matter growth rate $f$. From $E, P_{2,3}$ one can form consistency relations in terms of the HL functions. The simplest one is Eq. (21) or (22), expressed purely in terms of model-independent observables. If observations indicate a violation of a consistency relation, then the $\mathrm{DE}$ is not described by the HL in the quasi-static limit. Conversely, finding the predicted $k$-behavior would be a major confirmation of the scalar field picture of dark energy.

Non-linear effects will bring both more information and more unknowns into the picture so it is not clear how much, if at all, they would improve the task of reconstructing or rejecting the HL. The limitations of real-world observations, completely neglected here, are of course in practice the major hurdle on the path to this goal.

\section{ACKNOWLEDGMENTS}

It is a pleasure to thank Leonidas Christodoulou, Enrique Gaztañaga, Dragan Huterer, Eyal Kazin, Ofer Lahav and Shinji Tsujikawa for useful comments and conversations. We are grateful for the hospitality of the Centro de Sciencias de Benasque Pedro Pascual, where a part of this manuscript was prepared. The work of L.A. and I.S. is supported by the DFG through TRR33 "The Dark Universe". M.K. acknowledges funding by the Swiss National Science Foundation. M.M. is supported by CNPqBrazil. I.D.S. acknowledges STFC for financial support.

\section{Appendix A: Details of Horndeski Properties}

This appendix concerns the properties of the scalar-field theories described by the Horndeski Lagrangian given by the combination of terms presented in Eqs (16). The subscripts , $\phi$ and, $X$ denote derivation with respect to that variable. On a flat FRW background, the energy density and pressure are given by 


$$
\begin{aligned}
\rho_{x}= & 3 H^{2}\left(1-w_{1}\right)+2 X K_{, X}-K-2 X G_{3, \phi}+ \\
& +6 \dot{\phi} H\left(X G_{3, X}-G_{4, \phi}-2 X G_{4, \phi X}\right)+ \\
& +12 H^{2}\left(X\left(G_{4, X}+2 X G_{4, X X}\right)-G_{5, \phi}-X G_{5, \phi X}\right)+ \\
& +4 \dot{\phi} X H^{3}\left(G_{5, X}+X G_{5, X X}\right), \\
P_{x}= & -\left(3 H^{2}+2 \dot{H}\right)\left(1-w_{1}\right)+K-2 X G_{3, \phi}+4 X G_{4, \phi \phi}+ \\
& +2 \dot{\phi} H w_{1, \phi}-4 X^{2} H^{2} G_{5, \phi X}+2 \dot{\phi} X H^{3} G_{5, X}+\frac{\ddot{\phi}}{\dot{\phi}}\left(w_{2}-2 H w_{1}\right),
\end{aligned}
$$

where, given a slight rearrangement of the results in Refs [20, 21], we define four functions $w_{i}$ as

$$
\begin{aligned}
w_{1} \equiv & 1+2\left(G_{4}-2 X G_{4, X}+X G_{5, \phi}-\dot{\phi} X H G_{5, X}\right) \\
w_{2} \equiv & -2 \dot{\phi}\left(X G_{3, X}-G_{4, \phi}-2 X G_{4, \phi X}\right)+ \\
& +2 H\left(w_{1}-4 X\left(G_{4, X}+2 X G_{4, X X}-G_{5, \phi}-X G_{5, \phi X}\right)\right)- \\
& -2 \dot{\phi} X H^{2}\left(3 G_{5, X}+2 X G_{5, X X}\right), \\
w_{3} \equiv & 3 X\left(K, X+2 X K_{, X X}-2 G_{3, \phi}-2 X G_{3, \phi X}\right)+18 \dot{\phi} X H\left(2 G_{3, X}+X G_{3, X X}\right)- \\
& -18 \dot{\phi} H\left(G_{4, \phi}+5 X G_{4, \phi X}+2 X^{2} G_{4, \phi X X}\right)- \\
& -18 H^{2}\left(1+G_{4}-7 X G_{4, X}-16 X^{2} G_{4, X X}-4 X^{3} G_{4, X X X}\right)- \\
& -18 X H^{2}\left(6 G_{5, \phi}+9 X G_{5, \phi X}+2 X^{2} G_{5, \phi X X}\right)+ \\
& +6 \dot{\phi} X H^{3}\left(15 G_{5, X}+13 X G_{5, X X}+2 X^{2} G_{5, X X X}\right), \\
w_{4} \equiv & +2\left(G_{4}-X G_{5, \phi}-X G_{5, X} \ddot{\phi}\right) .
\end{aligned}
$$

All of the dynamics of linear perturbations are fully determined by the above four functions. In particular, the speed of propagation of gravitational waves, $c_{\mathrm{T}}$, and the normalization of the kinetic term of these tensor perturbations, $Q_{\mathrm{T}}$, is given by

$$
c_{\mathrm{T}}^{2}=\frac{w_{4}}{w_{1}}>0, \quad Q_{\mathrm{T}}=\frac{w_{1}}{4}>0,
$$

with positivity required by stability. From the above, is can be seen that $w_{1}$ has the meaning of the normalization of the tensor perturbations, i.e. it is the effective Planck mass squared. The corresponding quantities for the scalar degree of freedom, the sound speed of dark energy, $c_{\mathrm{s}}$, and the normalization of the kinetic energy for perturbations, $Q_{\mathrm{S}}$, in the presence of dust with energy density $\rho_{\mathrm{m}}$, are

$$
\begin{aligned}
c_{\mathrm{S}}^{2} & =\frac{3\left(2 w_{1}^{2} w_{2} H-w_{2}^{2} w_{4}+4 w_{1} w_{2} \dot{w}_{1}-2 w_{1}^{2}\left(\dot{w}_{2}+\rho_{\mathrm{m}}\right)\right)}{w_{1}\left(4 w_{1} w_{3}+9 w_{2}^{2}\right)}>0, \\
Q_{\mathrm{S}} & =\frac{w_{1}\left(4 w_{1} w_{3}+9 w_{2}^{2}\right)}{3 w_{2}^{2}}>0 .
\end{aligned}
$$

With above definitions in hand, we can define the five scale-independent functions $h_{1-5}$ which appeared in the result (17). All the observables for scalar perturbations in the quasi-static regime are determined by these five functions,

$$
\begin{aligned}
h_{1} & \equiv \frac{w_{4}}{w_{1}^{2}}=\frac{c_{\mathrm{T}}^{2}}{w_{1}}, \quad h_{2} \equiv \frac{w_{1}}{w_{4}}=c_{\mathrm{T}}^{-2} \\
h_{3} & \equiv \frac{H^{2}}{2 X M^{2}} \frac{2 w_{1}^{2} w_{2} H-w_{2}^{2} w_{4}+4 w_{1} w_{2} \dot{w}_{1}-2 w_{1}^{2}\left(\dot{w}_{2}+\rho_{\mathrm{m}}\right)}{2 w_{1}^{2}} \\
h_{4} & \equiv \frac{H^{2}}{2 X M^{2}} \frac{2 w_{1}^{2} H^{2}-w_{2} w_{4} H+2 w_{1} \dot{w}_{1} H+w_{2} \dot{w}_{1}-w_{1}\left(\dot{w}_{2}+\rho_{\mathrm{m}}\right)}{w_{1}}, \\
h_{5} & \equiv \frac{H^{2}}{2 X M^{2}} \frac{2 w_{1}^{2} H^{2}-w_{2} w_{4} H+4 w_{1} \dot{w}_{1} H+2 \dot{w}_{1}^{2}-w_{4}\left(\dot{w}_{2}+\rho_{\mathrm{m}}\right)}{w_{4}}
\end{aligned}
$$


and where the effective mass squared, $M^{2}$, can be expressed in terms of derivatives of the total pressure and total energy with respect to the scalar as

$$
M^{2}=\frac{3 H\left(P_{x, \phi}+\rho_{x, \phi}\right)+\dot{\rho}_{x, \phi}}{\dot{\phi}} .
$$

One may wonder whether it is possible to invert Eqs (A5) in order to obtain $\rho_{\mathrm{m}}$ as a function of the functions $h_{1-5}$. If this were possible, and if all the $h_{1-5}$ were observable, then one could measure $\Omega_{\mathrm{m} 0}$, contrary to our claim in the text. In a future paper we will discuss in detail the observability of the functions $h_{1-5}$ and we will show that in fact it is not possible to obtain $\Omega_{\mathrm{m} 0}$ this way. Here it will suffice to notice that $h_{1}$ is completely degenerate with $\Omega_{\mathrm{m} 0}$ (see Eq. (19)); it turns out that $\rho_{\mathrm{m}}$ is proportional to $1 / h_{1}$ and therefore the fact that $h_{1}$ can only be measured up to $\Omega_{\mathrm{m} 0}$ implies the same degeneracy in $\rho_{\mathrm{m}}$.

1] L. Amendola and S. Tsujikawa, Dark Energy: Theory and Observations. Cambridge University Press, 2010.

[2] T. Clifton, P. G. Ferreira, A. Padilla, and C. Skordis, "Modified Gravity and Cosmology,"

Phys.Rept. 513 (2012) 1-189, arXiv:1106.2476 [astro-ph.CO] .

[3] M. Kunz, "The phenomenological approach to modeling the dark energy," Comptes Rendus Physique 13 (2012) 539-565, arXiv:1204.5482 [astro-ph.CO].

[4] A. Stebbins, "Measuring Space-Time Geometry over the Ages," Int.J.Mod.Phys. D21 (2012) 1242017, arXiv:1205.4201 [gr-qc].

[5] A. Shafieloo and C. Clarkson, "Model independent tests of the standard cosmological model,"

Phys.Rev. D81 (2010) 083537, arXiv:0911.4858 [astro-ph.CO].

[6] A. Shafieloo and E. V. Linder, "Cosmographic Degeneracy," Phys.Rev. D84 (2011) 063519, arXiv:1107.1033 [astro-ph.CO].

[7] D. Huterer and G. Starkman, "Parameterization of dark-energy properties: A Principal-component approach," Phys.Rev.Lett. 90 (2003) 031301, arXiv:astro-ph/0207517 [astro-ph]

[8] W. Valkenburg, V. Marra, and C. Clarkson, "Testing the Copernican principle by constraining spatial homogeneity," arXiv:1209.4078 [astro-ph.CO]

[9] R. R. Caldwell and M. Doran, "Dark-energy evolution across the cosmological-constant boundary,"

Phys.Rev. D72 (2005) 043527, arXiv: astro-ph/0501104 [astro-ph].

[10] E. V. Linder, "Cosmic growth history and expansion history," Phys.Rev. D72 (2005) 043529, arXiv:astro-ph/0507263 [astro-ph].

[11] W. Hu and I. Sawicki, "A Parameterized Post-Friedmann Framework for Modified Gravity," Phys.Rev. D76 (2007) 104043, arXiv:0708.1190 [astro-ph].

[12] L. Amendola, M. Kunz, and D. Sapone, "Measuring the dark side (with weak lensing)," JCAP 0804 (2008) 013 , arXiv:0704.2421 [astro-ph].

[13] R. Bean and M. Tangmatitham, "Current constraints on the cosmic growth history," Phys.Rev. D81 (2010) 083534,
arXiv: 1002.4197 [astro-ph.CO]

[14] L. Pogosian, A. Silvestri, K. Koyama, and G.-B. Zhao, "How to optimally parametrize deviations from General Relativity in the evolution of cosmological perturbations?," Phys.Rev. D81 (2010) 104023, arXiv:1002.2382 [astro-ph.CO].

[15] E. Bertschinger, "On the Growth of Perturbations as a Test of Dark Energy," Astrophys.J. 648 (2006) 797-806, arXiv:astro-ph/0604485 [astro-ph].

[16] T. Baker, P. G. Ferreira, C. Skordis, and J. Zuntz, "Towards a fully consistent parameterization of modified gravity," Phys.Rev. D84 (2011) 124018, arXiv:1107.0491 [astro-ph.CO].

[17] R. A. Battye and J. A. Pearson, "Effective action approach to cosmological perturbations in dark energy and modified gravity," JCAP 1207 (2012) 019, arXiv:1203.0398 [hep-th].

[18] T. Baker, P. G. Ferreira, and C. Skordis, "The Parameterized Post-Friedmann Framework for Theories of Modified Gravity: Concepts, Formalism and Examples," arXiv:1209.2117 [astro-ph.CO] .

[19] P. Zhang, M. Liguori, R. Bean, and S. Dodelson, "Probing Gravity at Cosmological Scales by Measurements which Test the Relationship between Gravitational Lensing and Matter Overdensity," Phys.Rev.Lett. 99 (2007) 141302, arXiv:0704.1932 [astro-ph]

[20] A. De Felice and S. Tsujikawa, "Conditions for the cosmological viability of the most general scalar-tensor theories and their applications to extended Galileon dark energy models," JCAP 1202 (2012) 007, arXiv:1110.3878 [gr-qc].

[21] A. De Felice, T. Kobayashi, and S. Tsujikawa, "Effective gravitational couplings for cosmological perturbations in the most general scalar-tensor theories with second-order field equations,"

Phys.Lett. B706 (2011) 123-133, arXiv: 1108.4242 [gr-qc].

[22] V. Desjacques and R. K. Sheth, "Redshift space correlations and scale-dependent stochastic biasing of density peaks," Phys.Rev. D81 (2010) 023526, arXiv:0909.4544 [astro-ph.CO].

[23] R. P. Woodard, "Avoiding dark energy with $1 / R$ modifications of gravity," 
Lect.Notes Phys. 720 (2007) 403-433,

arXiv:astro-ph/0601672 [astro-ph].

[24] T. Chiba, " $1 / R$ gravity and scalar-tensor gravity," Phys.Lett. B575 (2003) 1-3, arXiv:astro-ph/0307338 [astro-ph].

[25] G. W. Horndeski, "Second-order scalar-tensor field equations in a four-dimensional space," Int.J.Th.Phys. 10 (1974) 363-384.

[26] C. Deffayet, X. Gao, D. Steer, and G. Zahariade, "From k-essence to generalised Galileons,"

Phys.Rev. D84 (2011) 064039, arXiv:1103.3260 [hep-th].

[27] C. Quercellini, L. Amendola, A. Balbi, P. Cabella, and M. Quartin, "Real-time Cosmology," arXiv:1011.2646 [astro-ph.CO].

[28] M. Kunz, "The dark degeneracy: On the number and nature of dark components," Phys.Rev. D80 (2009) 123001, arXiv:astro-ph/0702615 [astro-ph].

[29] N. Kaiser, "Clustering in real space and in redshift space," Mon.Not.Roy.Astron.Soc. 227 (1987) 1-27.

[30] S. Dodelson, Modern Cosmology. Academic Press, 2003.

[31] W. J. Percival and M. White, "Testing cosmological structure formation using redshift-space distortions," Mon.Not.Roy.Astron.Soc. 393 (2009) 297-308, arXiv:0808.0003 [astro-ph].

[32] E. Macaulay, H. A. Feldman, P. G. Ferreira, A. H. Jaffe, S. Agarwal, et al., "Power Spectrum Estimation from Peculiar Velocity Catalogues," Mon.Not.Roy.Astron.Soc. 425 (2012) 1709-1717, arXiv:1111.3338 [astro-ph.CO].

[33] A. Padilla and V. Sivanesan, "Covariant multi-galileons and their generalisation," arXiv:1210.4026 [gr-qc].

[34] I. Sawicki, I. D. Saltas, L. Amendola, and M. Kunz, "Consistent perturbations in an imperfect fluid," arXiv:1208.4855 [astro-ph.CO].

[35] T. Kobayashi, M. Yamaguchi, and J. Yokoyama, "Generalized G-inflation: Inflation with the most general second-order field equations," Prog.Theor.Phys. 126 (2011) 511-529, arXiv:1105.5723 [hep-th].

[36] R. Kimura and K. Yamamoto, "Constraints on general second-order scalar-tensor models from gravitational Cherenkov radiation," JCAP 1207 (2012) 050, arXiv:1112.4284 [astro-ph.CO]. 